\title{
DECONVOLUÇÃO DE EULER: PASSADO, PRESENTE E FUTURO - UM TUTORIAL
}

\author{
Valéria C. F. Barbosa e e João B. C. Silva² \\ Recebido em 20 junho, 2005 / Aceito em 05 dezembro, 2005 \\ Received on June 20, 2005 / Accepted on December 05, 2005
}

\begin{abstract}
In this tutorial we present a historical overview of the evolution of Euler deconvolution method consisting of three sections. In the first section, we recall the Euler deconvolution 2D and 3D classic formulation as a method to automatically locate sources of potential fields anomalies and point out the difficulties of this formulation: the presence of an undesirable spray of solutions, the empiric criteria used to determine the structural index (a parameter related with the source nature), the feasibility of applying Euler deconvolution to small ground data survey, and the determination of the dip and susceptibility contrast of geological contact (or the product of the susceptibility contrast and the thickness when applied to thin dike). In the second section, we present recent improvements aiming at minimizing some of the difficulties presented in the first section. They include: i) selecting the solutions essentially associated with observations presenting the largest signal-to-noise ratios; ii) using the correlation between the estimated anomaly baselevel and the observed anomaly itself or combining Euler deconvolution with the analytic signal to determine the structural index; iii) combining the results from (i) and (ii) to allow estimating the structural index independently of the number of solutions so that a small number of observations (such as in a ground survey) may be used; and iv) introducing additional equations, which are independent from Euler's equation to estimate the dip and susceptibility of 2D sources. In the third section we provide a short- to medium-term outlook into future developments. The main prospects are: i) furthering the improvements on the problems singled out in the second section; ii) developing methods to take vertical and lateral interferences into accounts; and iii) incorporating Euler deconvolution to constrain the source location estimates and use them in inversion methods to obtain a source delineation in a user-friendly computer environment.
\end{abstract}

Keywords: Euler Deconvolution, Automatic Potential Field Interpretations, Depth Estimation.

RESUMO. Neste tutorial apresentamos uma revisão da deconvolução de Euler que consiste de três partes. Na primeira parte, recordamos o papel da clássica formulação da deconvolução de Euler 2D e 3D como um método para localizar automaticamente fontes de campos potenciais anômalas e apontamos as dificuldades desta formulação: a presença de uma indesejável nuvem de soluções, o critério empírico usado para determinar o índice estrutural (um parâmetro relacionado com a natureza da fonte anômala), a exeqüibilidade da aplicação da deconvolução de Euler a levantamentos magnéticos terrestres, e a determinação do mergulho e do contraste de susceptibilidade magnética de contatos geológicos (ou o produto do contraste de susceptibilidade e a espessura quando aplicado a dique fino). Na segunda parte, apresentamos as recentes melhorias objetivando minimizar algumas dificuldades apresentadas na primeira parte deste tutorial. Entre estas melhorias incluem-se: i) a seleção das soluções essencialmente associadas com observações apresentando alta razão sinal-ruído; ii) 0 uso da correlação entre a estimativa do nível de base da anomalia e a própria anomalia observada ou a combinação da deconvolução de Euler com o sinal analítico para determinação do índice estrutural; iii) a combinação dos resultados de (i) e (ii), permitindo estimar o índice estrutural independentemente do número de soluções; desta forma, um menor número de observações (tal como em levantamentos terrestres) pode ser usado; iv) a introdução de equações adicionais independentes da equação de Euler que permitem estimar o mergulho e 0 contraste de susceptibilidade das fontes magnéticas 2D. Na terceira parte apresentaremos um prognóstico sobre futuros desenvolvimentos a curto e médio prazo envolvendo a deconvolução de Euler. As principais perspectivas são: i) novos ataques aos problemas selecionados na segunda parte deste tutorial; ii) desenvolvimento de métodos que permitam considerar interferências de fontes localizadas ao lado ou acima da fonte principal, e iii) uso das estimativas de localização da fonte anômala produzidas pela deconvolução de Euler como vínculos em métodos de inversão para obter a delineação das fontes em um ambiente computacional amigável.

Palavras-chave: Deconvolução de Euler, Interpretações Automática de Dados de Campos Potenciais, Estimativa de Profundidades.

\footnotetext{
${ }^{1}$ LNCC, Av. Getúlio Vargas, 333, Quitandinha, 25651-075 Petrópolis, Rio de Janeiro, Brasil. Tel: (24) 2233-6108; Fax: (24) 2231-5595 - E-mail: valcris@Incc.br.

2Universidade Federal do Pará, Dep. Geofísica, CG, Rua Augusto Corrêa, 1, Campus Universitário do Pará - 66017-970 Belém, Pará, Brasil. Fax: (91) 3201-1793 -E-mail: joaobcs@oi.com.br.
} 


\section{INTRODUÇÃO}

A partir de meados do século passado as agências governamentais tais como U.S. Geological Survey (USGS) e Canadian Geological Survey, iniciaram levantamentos aeromagnéticos sistemáticos de seus territórios. 0 resultado inicial foi uma vasta cobertura de dados magnéticos coletados ao longo de alguns milhões de linhas voadas, levando a um questionamento básico: como interpretar este imenso volume de dados? Este contexto impulsionou, a partir da década de 70, 0 desenvolvimento de métodos automáticos rápidos de interpretação de dados aeromagnéticos como, por exemplo, a deconvolução de Werner (Hartman et al., 1971), o método de Naudy (Naudy, 1971), o método CompuDepth (O'Brien, 1972), e a deconvolução de Euler (Thompson, 1982). Entre estes métodos automáticos destacam-se como os mais populares as deconvoluções de Werner e Euler que se assemelham fundamentalmente na eliminação da ambigüidade clássica da interpretação magnética envolvendo a estimação simultânea da magnetização e do volume das fontes causadoras. Essa ambigüidade é eliminada pela adoção de modelos interpretativos consistindo de fontes equivalentes simples como dipolos isolados, e linhas e superfícies de dipolos (ou monopolos). Na década de 90 a deconvolução de Euler tornou-se o mais empregado método de interpretação de dados aeromagnéticos.

Neste trabalho apresentamos uma evolução histórica da deconvolução de Euler: passado (formulação clássica e deficiências), presente (atual estado da arte e solução parcial de alguns problemas) e futuro (novas fronteiras).

\section{PASSADO: FORMULAÇÃO CLÁSSICA DA DECONVOLUÇÃO DE EULER}

\section{Teoria}

A anomalia magnética de campo total $T \equiv T(x, y, z)$ não corrigida de um campo regional aditivo constante e produzida por uma fonte pontual tridimensional (3D) situada nas coordenadas $x_{0}, y_{0}, z_{0}$ (referida a um sistema Cartesiano destral) satisfaz à equação homogênea de Euler 3D (Reid et al., 1990):

$$
\left(x-x_{0}\right) \frac{\partial}{\partial x} T+\left(y-y_{0}\right) \frac{\partial}{\partial y} T+\left(z-z_{0}\right) \frac{\partial}{\partial z} T=-\eta T
$$

em que $\eta$ é um parâmetro conhecido como índice estrutural, sendo uma medida da taxa de decaimento da anomalia magnética com a distância entre a fonte e o ponto de medida, i.e., um indicador da forma geométrica da fonte anômala (por exemplo, $\eta=0$ para um contato, $\eta=1$ para um dique vertical ou uma soleira, $\eta=2$ para um cilindro horizontal ou vertical e $\eta=3$ para uma esfera ou um dipolo). Presumindo-se o conhecimento preliminar do índice estrutural, a Eq. (1) pode ser expressa como

$$
\begin{aligned}
& x_{0} \frac{\partial h}{\partial x}+y_{0} \frac{\partial h}{\partial y}+z_{0} \frac{\partial h}{\partial z}+\eta b= \\
& =x \frac{\partial h}{\partial x}+y \frac{\partial h}{\partial y}+z \frac{\partial h}{\partial z}+\eta h
\end{aligned}
$$

em que $h \equiv h(x, y, z)=T(x, y, z)+b$ e $b$ um nível de base constante e desconhecido. Em notação matricial, a Eq. (2) pode ser reescrita como

$$
\mathbf{G} \mathbf{p}=\mathbf{y}
$$

sendo $\mathbf{p}$, 0 vetor de parâmetros desconhecidos, $\mathbf{G} \in R^{(N \times 4)}$ e $\mathbf{y} \in R^{N}$ definidos para cada janela móvel de dados com $N$ observações, levando a Eq. (3) à forma:

$$
\begin{gathered}
\left(\begin{array}{cccc}
\frac{\partial h_{1}}{\partial x} & \frac{\partial h_{1}}{\partial y} & \frac{\partial h_{1}}{\partial z} & \eta \\
\vdots & \vdots & \vdots & \vdots \\
\frac{\partial h_{N}}{\partial x} & \frac{\partial h_{N}}{\partial y} & \frac{\partial h_{N}}{\partial z} & \eta
\end{array}\right)\left(\begin{array}{c}
x_{0} \\
y_{0} \\
z_{0} \\
b
\end{array}\right) \\
=\left(\begin{array}{c}
x_{1} \frac{\partial h_{1}}{\partial x}+y_{1} \frac{\partial h_{1}}{\partial y}+z_{1} \frac{\partial h_{1}}{\partial z}+\eta h_{1} \\
\vdots \\
x_{N} \frac{\partial h_{N}}{\partial x}+y_{N} \frac{\partial h_{N}}{\partial y_{N}}+z_{N} \frac{\partial h_{N}}{\partial z}+\eta h_{N}
\end{array}\right)
\end{gathered}
$$

em que $\frac{\partial h_{i}}{\partial \varsigma}$ representa o gradiente de $h$ avaliado no i-ésimo ponto de observação em relação à variável $\varsigma$.

\section{Procedimentos Computacionais}

Para um valor presumivelmente conhecido $\eta$ e para cada posição de uma janela móvel de dados, a deconvolução de Euler estima as coordenadas de localização da fonte anômala $\left(x_{0}, y_{0}, z_{0}\right)$ e 0 nível de base ( $b$ ) resolvendo o sistema linear (3) de $N$ equações nestas 4 incógnitas via método dos mínimos quadrados, resultando no estimador

$$
\hat{p}=\left(\mathbf{G}^{T} \mathbf{G}\right)^{-1} \mathbf{G}^{T} \mathbf{y},
$$

em que $\hat{p}=\left(\begin{array}{llll}\hat{x}_{0} & \hat{y}_{0} & \hat{z}_{0} & \hat{b}\end{array}\right)^{T}$ é 0 vetor de estimativas dos parâmetros. Thompson (1982) recomendou aceitar apenas as estimativas $\hat{p}$ que satisfazem à inequação

$$
\frac{\hat{z}_{0}}{\eta \sigma_{z_{0}}}>\varepsilon
$$

em que $\sigma_{z_{0}}$ é 0 desvio padrão de $\hat{z}_{0}$ e $\varepsilon$ é um escalar positivo fornecido pelo intérprete. Cada estimativa aceita e relacionada com uma dada janela de dados é considerada como uma possível 
solução e um símbolo (representando o índice estrutural presumido) é plotado nas coordenadas $\left(\hat{x}_{0}, \hat{y}_{0}, \hat{z}_{0}\right)$. 0 índice estrutural tentativo que produz a menor dispersão das soluções é selecionado como a melhor estimativa do índice estrutural verdadeiro.

\section{Deficiências}

A formulação clássica da deconvolução de Euler apresenta duas fraquezas principais que são:

1) a presença de uma indesejável nuvem de soluções e

2) 0 critério empírico para estimar $\eta$ (tipo de fonte geológica), aceitando, como a melhor estimativa, o valor usado para $\eta$ que produz uma menor dispersão na nuvem de soluções aceitas.

Adicionalmente, há outras deficiências como por exemplo:

1) a inexeqüibilidade em levantamentos magnéticos terrestres com poucas observações;

2) a impossibilidade de determinação de outros parâmetros como por exemplo, a susceptibilidade magnética e o mergulho das fontes magnéticas;

3) a ineficácia em ambientes complexos consistindo de múltiplos corpos interferentes laterais e verticais dentro de uma mesma janela e

4) a impossibilidade de integração das soluções da deconvolução de Euler com a modelagem geológica 2D ou 3D.

\section{PRESENTE: 0 ATUAL ESTADO-DA-ARTE COM ÊNFASE NAS RECENTES MELHORIAS DA DECONVOLUÇÃO DE EULER}

A partir da década de 90 esforços têm sido feitos no sentido de eliminar, ou pelo menos, reduzir as desvantagens da deconvolução de Euler citadas acima. Apresentamos, a seguir, uma análise destes esforços.

\section{Redução da nuvem de soluções}

Thompson (1982) recomendou aceitar apenas as soluções que satisfazem a inequação (6). Barbosa et al. (1999) mostraram que este critério tende a selecionar soluções estimadas pelas janelas móveis situadas nas proximidades do pico da anomalia porque nesta região há 0 decréscimo de $\sigma_{z_{0}}$ (desvio padrão de $\hat{z}_{0}$ ) associado ao aumento dos valores singulares da matriz $\mathbf{G}$, implicando a diminuição de $\sigma_{z 0}$. Este critério, no entanto, é fortemente dependente da escolha do valor de $\epsilon$ [inequação (6)] fornecido pelo interprete e o resultado é, em geral, uma indesejável nuvem de dispersão das soluções estimadas via deconvolução de Euler que pode chegar à ordem de $10^{3}$ (no caso 3D), dificultando a interpretação dos resultados. Portanto, uma substancial redução da nuvem de dispersão das soluções estimadas é fundamental para viabilizar a aplicação da deconvolução de Euler a grandes levantamentos aeromagnéticos contendo um elevado número de observações. Com esse intuito, Fairhead et al. (1994) reduziram o número de soluções estimadas selecionando apenas aquelas associadas a janelas móveis de dados contendo valores absolutos grandes para os gradientes horizontais e vertical. Para tanto 0 método de Fairhead et al. (1994) requer a redução da anomalia magnética ao pólo. Barbosa et al. (1999), por sua vez, reduziram o número de soluções selecionando apenas aquelas que satisfazem a inequação (6) e que, também, obedecem à inequação

$$
\sqrt{\|\mathbf{y}-\mathbf{G p}\|^{2} / N}<\gamma,
$$

em que $\gamma$ é o menor escalar positivo produzindo vetores $\mathbf{y}$ e $\mathbf{G p}$ suficientemente próximos entre si. Mikhailov et al. (2003) desenvolveram uma técnica de análise de "clusters" baseada em uma abordagem topológica e geométrica para estudar e reduzir as concentrações das soluções. Finalmente, Silva \& Barbosa (2003) deduziram os estimadores analíticos para as posições horizontais e vertical de uma fonte anômala magnética 3D e, analisando o comportamento destes estimadores como uma função das coordenadas $x, y$ e $z$ das observações, lançaram os fundamentos teóricos para a escolha das melhores soluções estimadas via deconvolução de Euler tendo como base o delineamento da área plana das estimativas $\hat{x}_{0}$ e $\hat{y}_{0}$ onde são produzidas estimativas consistentes da localização da fonte anômala.

Ressalte-se que, uma vez que a nuvem de soluções é causada primordialmente pela instabilidade da solução na presença de ruído, a maioria das tentativas de redução da nuvem de soluções baseia-se essencialmente em obter as soluções associadas com a maior razão sinal/ruído.

\section{Critério para estimar 0 índice estrutural $(\eta)$}

A deconvolução de Euler tem a potencial vantagem de não necessitar a postulação de um modelo interpretativo. No entanto, como mostra a Eq. (2) esta vantagem potencial requer o conhecimento a priori do parâmetro $\eta$ (i.e., da geometria da fonte) para estimar corretamente as coordenadas de localização da fonte anômala. De fato, a escolha do índice estrutural correto tem sido a principal dificuldade na aplicação da deconvolução de Euler (Reid, 1995). 
Thompson (1982) apresentou um critério empírico para estimar $\eta$, aceitando, como a melhor estimativa, o valor usado para $\eta$ que produz uma menor dispersão na nuvem de soluções. Esta estimativa ad hoc de $\eta$, foi adaptada para 0 caso 3D por Reid et al. (1990) e apesar de ser amplamente utilizada tem sido fortemente questionada por alguns autores (Ravat, 1996; Reid, 1995) devido a resultados insatisfatórios. Barbosa et al. (1999) mostraram numericamente que este critério além de empírico pode levar a erros na estimativa de $\eta$. Silva et al. (2001) mostraram analiticamente que esse critério é teoricamente exato i.e., quando aplicado a dados sem ruído, a dispersão das soluções da deconvolução de Euler é, de fato devida, exclusivamente, ao uso errado do índice estrutural; ele porém falha em casos práticos porque o ruído nos dados também contribui para a dispersão das soluções.

Barbosa et al. (1999) propuseram um novo método para estimar $\eta$ que foi deduzido teoricamente da equação homogênea de Euler e baseia-se na correlação entre a anomalia de campo total e as estimativas do nível de base ( $b$ ) computadas no centro de cada janela móvel de dados usando-se diferentes valores tentativos de $\eta$. 0 valor do índice estrutural que produz a menor correlação é tomado como a estimativa de $\eta$. Portanto, ao contrário do critério de Thompson (1982), o método de Barbosa et al. (1999) para estimar $\eta$ não depende das soluções estimadas. Computacionalmente, este método é muito simples; porém, no caso de múltiplas fontes com diferentes formas geométricas, a correlação mínima entre $h$ e $b$ deve ser calculada para cada fonte magnética, 0 que dificulta um processamento totalmente automático.

Recentemente, Salem \& Ravat (2003) apresentaram um novo método denominado "AN-EUL" baseado na combinação da deconvolução de Euler com o método do sinal analítico ( $\mathrm{Na}$ bighian, 1972), que permite estimar 0 índice estrutural e a profundidade da fonte anômala na coordenada de máxima amplitude $\left(x_{0}, y_{0}\right)$ do sinal analítico (AAS), respectivamente, através das equações:

$$
\eta=\left(\frac{2\left|A A S_{1}\right|^{2}-\left|A A S_{2} \| A A S_{0}\right|}{\left|A A S_{2} \| A A S_{0}\right|-\left|A A S_{1}\right|^{2}}\right)_{x=x_{0}, y=y_{0}},
$$

$$
\hat{z}_{0}=\left(\frac{\left|A A S_{1} \| A A S_{0}\right|}{\left|A A S_{2} \| A A S_{0}\right|-\left|A A S_{1}\right|^{2}}\right)_{x=x_{0}, y=y_{0}},
$$

em que $\left|A A S_{n}(x, y)\right|$ é a amplitude de $n$-ésima ordem do sinal analítico (Debeglia \& Corpel, 1997) sendo expressa por

$$
\begin{gathered}
\left|A A S_{n}(x, y)\right|= \\
=\sqrt{\left(\frac{\partial T_{n}^{z}}{\partial x}\right)^{2}+\left(\frac{\partial T_{n}^{z}}{\partial y}\right)^{2}+\left(\frac{\partial T_{n}^{z}}{\partial z}\right)^{2}},
\end{gathered}
$$

e $T_{n}^{z}$ é a derivada vertical de ordem $n$ da anomalia de campo total. Este método requer o conhecimento a priori das coordenadas horizontais $\left(x_{0}, y_{0}\right)$ de localização da fonte magnética, uma vez que as Eqs. (8) e (9) usam as amplitudes do sinal analítico avaliadas nestas coordenadas. 0 método AN-EUL estima tais coordenadas como os maximantes da família de funções $A A S_{n}(x, y)$. Uma desvantagem do método AN-EUL é o cálculo de derivadas até de terceira ordem da anomalia de campo total, o que é, matematicamente, uma operação instável produzindo amplificação do ruído contido nos dados observados. 0 método AN-EUL contorna este problema computando a continuação para cima da anomalia de campo total. Esta transformação é estável, mas leva à perda de resolução, o que pode ser catastrófico, principalmente, para a estimativa da coordenada vertical $\left(\hat{z}_{0}\right)$ de localização da fonte anômala. Além disso, a continuação para cima acentua a interferência de anomalias vizinhas. Adicionalmente, vale ressaltar que o método AN-EUL também não permite um processamento automático para computar a localização e geometria da fonte anômala. Portanto, no caso de múltiplas fontes as Eqs. (8) e (9) devem ser calculadas para cada fonte magnética.

\section{Exequiibilidade em levantamentos magnéticos terrestres}

A formulação convencional da deconvolução de Euler tornou este método inexeqüivel em levantamentos terrestres com poucas observações. Isto porque 0 critério de menor dispersão das soluções para a escolha ad hoc de $\eta$ (Thompson, 1982) exige um número grande de soluções estimadas e, como cada solução estimada está relacionada a uma janela móvel de dados, um número grande de soluções demanda um número grande de observações magnéticas. Vale então notar que o que tornava proibitiva a aplicação da deconvolução de Euler a levantamentos terrestres era o critério empírico de Thompson (1982) para estimar $\eta$. Barbosa et al. (2000) mostraram a viabilidade de aplicação da deconvolução de Euler a levantamentos com um número limitado de observações como os levantamentos magnéticos terrestres. Para tanto os autores empregaram o método de mínima correlação entre a anomalia de campo total e as estimativas do nível de base para a determinação de $\eta$ (Barbosa et al., 1999). Similarmente, Salem \& Ravat (2003) ressaltaram a exeqüibilidade 
de aplicar o método AN-EUL a levantamentos magnéticos terrestres porque este método, ao contrário do critério de Thompson (1982), estima o índice estrutural [V. Eq. (8)] independentemente do número de soluções estimadas.

\section{Determinação de outros parâmetros}

A formulação clássica da deconvolução de Euler permite estimar apenas as coordenadas de localização da fonte anômala $\left(x_{0}, y_{0}, z_{0}\right)$ e 0 nível de base $(b)$. Mushayandebvu et al. (2001) introduziram uma segunda equação que permitiu a determinação do contraste de susceptibilidade e do mergulho de fontes magnéticas 2D. Especificamente, para 0 caso do contato magnético, cujo índice estrutural é zero, os autores resolvem as seguintes equações:

$$
x_{0} \frac{\partial T}{\partial x}+z_{0} \frac{\partial T}{\partial z}+A_{1}=x \frac{\partial T}{\partial x}+z \frac{\partial T}{\partial z}
$$

e

$$
x_{0} \frac{\partial T}{\partial z}-z_{0} \frac{\partial T}{\partial x}+A_{2}=x \frac{\partial T}{\partial z}-z \frac{\partial T}{\partial x}
$$

em que

$$
A_{1}=\alpha \operatorname{sen}(\beta)
$$

e

$$
A_{2}=\alpha \cos (\beta),
$$

sendo $\beta=2 I-d-90^{\circ}$ e $\alpha=2 k F c \operatorname{sen}(d)$, sendo $d 0$ mergulho do contato, $k 0$ contraste de susceptibilidade magnética, $F$ a intensidade magnética do campo da Terra, $c=$ $1-\cos ^{2}(i) \operatorname{sen}^{2}(D)$, em que $i$ e $D$ são, respectivamente, a inclinação e 0 azimute do campo geomagnético em relação a $x$ e $\tan (I)=\tan (i) / \cos (D)$. Em notação matricial a Eq. (11) pode ser expressa como:

$$
\mathbf{G}_{1} \mathbf{p}_{1}=\mathbf{y}_{1},
$$

sendo $\mathbf{p}_{1}, 0$ vetor de parâmetros desconhecidos, $\mathbf{G}_{1} \in R^{(N \times 3)}$ e $\mathbf{y}_{1} \in R^{N}$ definidos para cada janela móvel de dados com $N$ observações, levando a Eq. (15) à forma:

$$
\left(\begin{array}{ccc}
\frac{\partial h_{1}}{\partial x} & \frac{\partial h_{1}}{\partial z} & 1 \\
\vdots & \vdots & \vdots \\
\frac{\partial h_{N}}{\partial x} & \frac{\partial h_{N}}{\partial z} & 1
\end{array}\right)\left(\begin{array}{c}
x_{0} \\
z_{0} \\
A_{1}
\end{array}\right)=\left(\begin{array}{c}
x_{1} \frac{\partial h_{1}}{\partial x}+z_{1} \frac{\partial h_{1}}{\partial z} \\
\vdots \\
x_{N} \frac{\partial h_{N}}{\partial x}+z_{N} \frac{\partial h_{N}}{\partial z}
\end{array}\right)
$$

Similarmente, em notação matricial, a Eq. (12) pode ser expressa como:

$$
\mathbf{G}_{2} \mathbf{p}_{2}=\mathbf{y}_{2},
$$

sendo $\mathbf{p}_{2}$, o vetor de parâmetros desconhecidos, $\mathbf{G}_{2} \in R^{(N \times 3)}$ e $\mathbf{y}_{2} \in R^{N}$ definidos para cada janela móvel de dados com $N$ observações, levando a Eq. (17) à forma:

$$
\left(\begin{array}{ccc}
\frac{\partial h_{1}}{\partial x} & -\frac{\partial h_{1}}{\partial z} & 1 \\
\vdots & \vdots & \vdots \\
\frac{\partial h_{N}}{\partial x} & -\frac{\partial h_{N}}{\partial z} & 1
\end{array}\right)\left(\begin{array}{c}
x_{0} \\
z_{0} \\
A_{2}
\end{array}\right)=\left(\begin{array}{c}
x_{1} \frac{\partial h_{1}}{\partial x}-z_{1} \frac{\partial h_{1}}{\partial z} \\
\vdots \\
x_{N} \frac{\partial h_{N}}{\partial x}-z_{N} \frac{\partial h_{N}}{\partial z}
\end{array}\right)
$$

Computacionalmente, os autores resolvem as Eqs. (15) e (17) via método dos mínimos quadrados para estimar simultaneamente as coordenadas de localização $\left(x_{0} \mathrm{e} z_{0}\right)$ e, independentemente, os parâmetros $A_{1}$ e $A_{2}$. Posteriormente, as estimativas de $A_{1}$ e $A_{2}$ são usadas para estimar as variáveis desconhecidas $\alpha$ e $\beta$ através das Eqs. (13) e (14), respectivamente. Presumindose 0 conhecimento a priori do vetor campo geomagnético e supondo magnetização induzida apenas, determina-se, a partir das variáveis $\alpha$ e $\beta$, o ângulo de mergulho ( $d$ ) e 0 contraste de susceptibilidade $(k)$ do contato magnético. Esta metodologia foi denominada pelos autores de "deconvolução de Euler estendida", que também foi desenvolvida para diques finos para estimar 0 ângulo de mergulho e o produto do contraste de susceptibilidade e a espessura do dique.

Vale ressaltar que tanto a deconvolução de Euler clássica como a estendida eliminam a ambigüidade fundamental da geofísica envolvendo o produto propriedade física $\times$ volume, porém de formas diferentes. A deconvolução de Euler clássica e suas modificações pressupõem fontes pontuais e estimam as coordenadas de localização e a geometria da fonte magnética (i.e., $\eta$ ), mas não estimam a propriedade física. Ao contrário, a deconvolução de Euler estendida pressupõe o conhecimento da geometria da fonte magnética (i.e., $\eta=0$ para contato geológico e $\eta=1$ para dique fino) para estimar a propriedade física (contraste de susceptibilidade no caso do contato e o produto do contraste de susceptibilidade pela espessura no caso do dique), mergulho e coordenadas de localização desta fonte. Computacionalmente, a deconvolução de Euler estendida é bastante simples podendo ser aplicada a ambientes com várias fontes $2 \mathrm{D}$ distantes entre si e a escolha da geometria da fonte (contado ou dique fino) é realizada através da menor dispersão das soluções selecionadas, tal como a versão clássica da deconvolução de Euler. A diferença é que a análise de dispersão na deconvolução estendida é realizada para todos os parâmetros, i.e., $x_{0}, z_{0}, k$ e $d$.

Recentemente, Mushayandebvu et al. (2004) empregaram a deconvolução de Euler estendida a corpos 2D (diques ou contatos) detectados em uma interpretação 3D. Para tanto, os autores partem da deconvolução de Euler convencional e detectam 0 autovetor associado ao autovalor próximo a zero da matriz $\mathbf{G}^{T} \mathbf{G}$ 
associada, no plano $x y$, à direção do corpo 2D. Finalmente, uma vez detectadas as direções dos corpos 2D, os autores empregam, para cada um deles a deconvolução de Euler estendida objetivando estimar 0 ângulo de mergulho e o contraste de susceptibilidade.

\section{Eficiência em ambientes complexos com múltiplos corpos}

Matematicamente, o sistema de equações lineares (3) mostra que um conjunto de dados magnéticos pode estimar apenas uma fonte anômala localizada nas coordenadas $x_{0}, y_{0} \mathrm{e} z_{0}$. Portanto, teoricamente, a deconvolução de Euler convencional parte do princípio que cada janela móvel de dados é capaz de estimar uma única fonte magnética. No entanto, na prática, devido a ruído e outras incertezas, há a necessidade que várias janelas móveis detectem uma única fonte magnética. Esta limitação torna impraticável 0 uso da deconvolução de Euler clássica na interpretação de ambientes complexos em que uma mesma janela móvel de dados registra a reposta de mais de uma fonte magnética. Hansen \& Suciu (2002) desenvolveram a generalização da deconvolução de Euler para múltiplas fontes permitindo a estimativa das coordenadas de localização de várias fontes magnéticas dentro de uma mesma janela móvel de dados. A formulação matemática deste método baseia-se na expressão tensorial da equação de Euler levando a um sistema não linear de equações. Como a solução de um sistema não linear demanda um razoável esforço computacional, os autores propõem uma estratégia que recai na solução de um sistema de equações lineares sobredeterminado. Hansen \& Suciu (2002) afirmam que 0 algoritmo é notoriamente complicado devido à presença de tensores e apontam duas cruciais desvantagens: (1) a restrição que todas as fontes dentro de uma dada janela devem ter o mesmo índice estrutural (i.e., a mesma geometria) e; (2) o significante esforço computacional.

\section{Integração das soluções da deconvolução de Euler dentro de modelos geológicos 2D ou 3D}

Uma das grandes vantagens da deconvolução de Euler clássica é fornecer uma rápida estimativa da localização e da geometria das fontes anômalas a partir de dados potenciais, sem a necessidade de presumir a direção do vetor magnetização. Na prática, a deconvolução de Euler é usada para gerar um complexo "spray" de profundidades do topo ou do centro das fontes anômalas, na maioria das vezes de difícil entendimento para os geólogos. Recentemente, Guillen et al. (2004) usaram a solução estimada pela deconvolução de Euler como vínculo em um método de inversão visando a construção de um ambiente geológico interpretativo 2D e 3D.

Enfatizamos que a principal vantagem da integração das soluções da deconvolução de Euler dentro de modelos geológicos é a facilidade da visualização espacial do resultado estimado em um ambiente geológico. Apesar desta facilidade atender a demanda dos geólogos, enfatizamos que o ônus desta abordagem é a perda da velocidade de processamento, uma vez que, para produzir 0 delineamento da geometria das fontes, é necessário 0 emprego de algoritmos de inversão.

\section{Outras contribuições relevantes}

Recentemente, a deconvolução de Euler recebeu especial atenção em seus aspectos teóricos, deixando de ser um método com alguns aspectos práticos empíricos. Neste contexto, alguns autores contribuíram para o entendimento da deconvolução de Euler e, de fato, torná-lo um método muito além de uma "caixa preta". Barbosa et al. (1999), Silva et al. (2001) e Silva \& Barbosa (2003) contribuíram na dedução analítica dos estimadores de $x_{0}, y_{0}, z_{0}$ e $b$, no estudo numérico da estabilidade, unicidade e tendenciosidade das soluções estimadas e suas propriedades de simetria e espalhamento. Zhang et al. (2000) desenvolveram e modificaram a deconvolução de Euler convencional para dados do tensor gradiente gravimétrico. Nabighian \& Hansen (2001) mostraram que a deconvolução de Euler estendida (Mushayandebvu et al., 2001) leva a uma generalização e unificação com a deconvolução de Werner e provaram, via transformada de Hilbert generalizada, que o método de Mushayandebvu et al. (2001) pode ser estendido para o caso 3D. Estes trabalhos, apesar de teóricos, não são simples preciosismos matemáticos, mas fundamentos sólidos para formulação e implementação de métodos práticos.

Adicionalmente, assistimos nos últimos anos a uma verdadeira torrente de aplicações da deconvolução de Euler nas mais diferentes áreas como por exemplo: geofísica arqueológica (Murdie et al., 1999), estudo de crateras de impacto (Forsyth et al., 1990; Keating, 1998) e geofísica ambiental (Ravat et al., 1996).

\section{FUTURO: NOVAS FRONTEIRAS DA DECONVOLUÇÃO DE EULER}

0 futuro imediato da deconvolução de Euler será a continuidade e aperfeiçoamento da maioria das técnicas acima citadas para solucionar as deficiências ainda presentes no método. Apesar dos esforços realizados, a estimativa automática e rápida do índice estrutural em ambientes geológicos complexos com fontes geológicas interferentes, tanto verticais como laterais, permanece 
um problema sem solução. Porém, o grande desafio será atender ao público de geólogos que demanda uma interpretação visualmente identificável com um ambiente geológico. Neste sentido, a integração das soluções da deconvolução de Euler com modelos 3D é uma linha de pesquisa que hoje representa a nova fronteira relacionada a este método. No entanto, esta integração implicará um radical distanciamento da filosofia inicial do método da deconvolução de Euler que é o fornecimento de uma interpretação automática e rápida. Isto porque, para a construção de um ambiente geológico 3D, a solução da deconvolução de Euler deverá ser usada como vínculo em um processo de inversão, implicando, assim uma interpretação computacionalmente dispendiosa e lenta. Por outro lado, apesar destas desvantagens a interpretação geológica 3D associada a técnicas de visualização 3D será, incontestavelmente, uma realidade demandada para 0 início deste novo milênio.

\section{CONCLUSÕES}

A deconvolução de Euler é um método de interpretação automática que fornece uma estimativa da posição horizontal e da profundidade de fontes anômalas a partir de dados de campos potenciais. Originalmente, este método foi amplamente usado para interpretar um grande volume de dados aeromagnéticos devido à alta velocidade de processamento, e à desnecessidade dos conhecimentos da geometria da fonte geológica anômala e do vetor magnetização das fontes. No entanto, a formulação original deste método apresentou duas grandes desvantagens:

i) a presença de uma enorme quantidade de possíveis soluções e

ii) o critério empírico e ineficiente para estimar tipo de fonte geológica.

A partir do início da década de 90 a deconvolução de Euler viveu uma fenomenal popularidade tornando-se a mais conhecida técnica de interpretação de dados aeromagnéticos, familiar tanto aos geofísicos como aos geólogos. Uma importante explicação para esta popularidade foi a implementação deste método em ambiente amigável pela indústria de software, 0 que 0 consagrou como uma das mais famosas técnicas geofísicas lacrada em uma "caixa preta". A partir do final da década de 90 iniciouse a fase de amadurecimento da deconvolução de Euler direcionada ao seu entendimento teórico, resultando na solução de algumas de suas deficiências e em extensões metodológicas. Entre as deficiências solucionadas e em via de solução, citamos: 1) a redução da indesejável nuvem de soluções e 2) 0 desenvolvimento de critérios matemáticos para estimar o índice estrutural (tipo de fonte geológica) no caso de anomalias isoladas. Entre as extensões metodológicas destacamos a deconvolução de Euler estendida para determinar o mergulho e 0 contraste de susceptibilidade de contatos geológicos ou o produto do contraste de susceptibilidade pela espessura no caso de um dique fino.

Recentemente, assistimos à transformação de uma técnica originalmente dominada por aspectos práticos empíricos e pertencente ao reino das "caixas pretas" em um MÉTODO de fato.

0 que 0 futuro reserva para a deconvolução de Euler? Certamente, reserva a sua integração com a inversão para a geração de imagens de ambientes geológicos 2D e 3D.

\section{AGRADECIMENTOS}

Os autores agradecem ao Editor Cleverson G. Silva e ao revisor anônimo pelas correções realizadas ao manuscrito original. Os autores também agradecem ao apoio financeiro recebido através do CNPq (bolsas de Produtividade em Pesquisa) e ao Dr. CarIos A. Mendonça pelo incentivo inicial na elaboração deste trabalho. Este trabalho teve apoio financeiro do CNPq pelos projetos definidos nos contratos: 471168/2004-1, 505265/2004-4 e 504419/2004-8. Um dos autores (V.C.F.B.) também recebeu apoio de projetos de pesquisas financiados pelo CNPq (contrato No. 472229/03-6) e pelo CNPq/FAPERJ (contrato No. E26/170.733/2004).

\section{REFERÊNCIAS}

BARBOSA VCF, SILVA JBC \& MEDEIROS WE. 1999. Stability analysis and improvement of structural index estimation in Euler deconvolution. Geophysics, 64: 48-60.

BARBOSA VCF, SILVA JBC \& MEDEIROS WE. 2000. Making Euler deconvolution applicable to small ground magnetic surveys. Journal of Applied Geophysics, 43: 55-68.

DEBEGLIA N \& CORPEL J. 1997. Automatic 3-D interpretation of potential field data using analytic signal derivatives. Geophysics, 62: 87-96.

FAIRHEAD JD, BENNETT KJ, GORDON DRH \& HUANG D. 1994. Euler: Beyond the "Black Box". In Anais 64th Annual International Meeting, Society of Exploration Geophysicists. 422-424, Los Angeles - California.

FORSYTH DA, PILKINGTON M, GRIEVE RAF \& ABINETT D. 1990. A major circular structure beneath southern Lake Huron from potential field data. Geology, 18: 773-777.

GUILLEN A, COURRIOUX G, CALCAGNO P, FITZGERALD D, LEES T \& MCINERNEY P. 2004. The BRGM 3DWEG gravity /magnetic constrained inversion applied to Broken Hill. In ASEG Conference Proceedings Sydney. 
HANSEN RO \& SUCIU L. 2002. Multiple-source Euler deconvolution. Geophysics, 67: 525-535.

HARTMAN RR, TESKEY DJ \& FRIEDBERG JL. 1971. A system for rapid digital aeromagnetic interpretation. Geophysics, 36: 891-918.

KEATING PB. 1998. Weighted Euler deconvolution of gravity data. Geophysics, 63: 1595-1603.

MIKHAILOV V, GALDEANO A, DIAMENT M, GVISHIANI A, AGAYAN S, BOBOUTDINOV S, GRAEVA E \& SAILHAC P. 2003. Application of artificial intelligence for Euler solutions clustering. Geophysics, 68: 168-180.

MURDIE RE, STYLES P, UPTON P, EARDLEY P \& CASSIDY NJ. 1999. Euler Deconvolution methods used to determine the depth to archaeological features, Geoarchaeology, Geological Society Special publication 153: $35-40$

MUSHAYANDEBVU MF, VAN DRIEL P, REID AB \& FAIRHEAD JD. 2001. Magnetic source parameters of two-dimensional structures using extended Euler deconvolution. Geophysics, 66: 814-823.

MUSHAYANDEBVU MF, LESUR VP, REID AB \& FAIRHEAD JD. 2004. Grid Euler deconvolution with constraints for 2D structures. Geophysics, 69 : 489-496.

NABIGHIAN MN. 1972. The analytic signal of two-dimensional magnetic bodies with polygonal cross-section: Its properties and use for automated anomaly interpretation. Geophysics, 37: 507-517.

NABIGHIAN MN \& HANSEN RO. 2001. Unification of Euler and Werner deconvolution in three dimensions via the generalized Hilbert transform. Geophysics, 66: 1805-1810.

NAUDY H. 1971. Automatic determination of depth on aeromagnetic profiles. Geophysics, 36: 717-722.
O'BRIEN DP. 1972. CompuDepth - A new method for depth-to-basement calculation: In $42^{\text {nd }}$ Annual International Meeting, Society of Exploration Geophysicists, Anaheim, California.

RAVAT D. 1996. Analysis of the Euler method and its applicability in environmental magnetic investigations. Journal of Environmental and Engineering Geophysics, 1: 229-238.

REID AB, ALLSOP JM, GRANSER H, MILLETT AJ \& SOMERTON IW. 1990. Magnetic interpretation in three dimensions using Euler deconvolution. Geophysics, 55: 80-91.

REID AB. 1995. Euler deconvolution: past, present and future - a review. In Anais 65th Annual International Meeting 65th Society of Exploration Geophysicists, 272-273, Houston, Texas.

SALEM AED \& RAVAT D. 2003. A combined analytic signal and Euler method (AN-EUL) for automatic interpretation of magnetic data. Geophysics, 68: 1952-1961.

SILVA JBC, BARBOSA VCF \& MEDEIROS WE. 2001. Scattering, symmetry, and bias analysis of source position estimates in Euler deconvolution and its practical implications. Geophysics, 66: 1149-1156.

SILVA JBC \& BARBOSA VCF. 2003. 3D Euler deconvolution: Theoretical basis for automatically selecting good solutions. Geophysics, 68: 1962-1968.

THOMPSON DT. 1982. EULDPH: A new technique for making computerassisted depth estimates from magnetic data. Geophysics, 47: 31-37.

ZHANG C, MUSHAYANDEBVU MF, REID AB, FAIRHEAD JD \& ODEGARD

ME. 2000. Euler deconvolution of tensor gradient gravity data. Geophysics, 65: 512-520.

\section{NOTAS SOBRE OS AUTORES}

Valéria C. F. Barbosa é graduada em Geologia (UFRRJ-1986), Mestre (1991) e Doutora (1998) em Geofísica pela UFPA. Trabalhou como geóloga na empresa Geopetro Consult. e Geofísica Ltda (1987-1989). É pesquisadora no LNCC desde 1998 e desde 2001 é pesquisadora do CNPq. Colabora como professora e orientadora no ON desde 2003. Em 2002 e 2004 ganhou da SEG (Society Exploration of Geophysicists) os prêmios de Menção Honrosa na categoria Best Paper in GEOPHYSICS e de "Outstanding GEOPHYSICS Reviewer", respectivamente. Atua como coordenadora dos seguintes projetos: EDITAL UNIVERSAL (CNPq) e PRIMEIROS PROJETOS (FAPERJ). Atua como vice-coordenadora dos seguintes projetos: CTMINERAL e CTPETRO. É editora associada da revista GEOPHYSICS (SEG) desde 2005.

João B. C. Silva é graduado em Geologia (UFRJ-1972), Mestre em Geofísica (UFPA-1976) e PhD em Geofísica (University of Utah-1982). Professor assistente (1974-1977), professor associado (1977-1997), e professor titular desde 1997 no Departamento de Geofísica da UFPA. Pesquisador visitante no LNCC (1998-1999). Em 1999, recebeu o prêmio "Geofísico de Destaque na área de mineração" da SBGf. Em 1999 e 2004 ganhou da SEG (Society Exploration of Geophysicists) os prêmios de "Outstanding GEOPHYSICS reviewer" e de "Outstanding GEOPHYSICS Associate Editor", respectivamente. Em 2002 recebeu da SEG o prêmio de Menção Honrosa na categoria Best Paper in GEOPHYSICS. É Coordenador da Pós-graduação em Geofísica da UFPA. Atuou como consultor da CAPES e no CA-GM do CNPq. Atua como coordenador dos seguintes projetos: EDITAL UNIVERSAL (CNPq), CTMINERAL e CTPETRO. Editor associado da revista GEOPHYSICS SEG (1999-2005). 